\section{Histone-induced thrombotic thrombocytopenic purpura in adamts13 zebrafish depends on von Willebrand factor}

\author{
Liang Zheng, ${ }^{1}$ Mohammad S. Abdelgawwad, ${ }^{1}$ Di Zhang, ${ }^{1}$ Leimeng $\mathrm{Xu},{ }^{1}$ Shi \\ Wei, ${ }^{2}$ Wenjing $\mathrm{CaO}^{1}$ and X. Long Zheng ${ }^{1}$
}

Divisions of ${ }^{1}$ Laboratory Medicine and ${ }^{2}$ Anatomic Pathology, Department of Pathology, The University of Alabama at Birmingham, Birmingham, AL, USA

\section{ABSTRACT}

T hrombotic thrombocytopenic purpura (TTP) is caused by severe deficiency of ADAMTS13 (A13), a plasma metalloprotease that cleaves endothelium-derived von Willebrand factor (VWF). However, severe A13 deficiency alone is often not sufficient to cause an acute TTP; additional factors may be required to trigger the disease. Using CRISPR/Cas9, we created and characterized several novel zebrafish lines carrying a null mutation in $a 13^{-1}, v w f$, and both. We further used these zebrafish lines to test the hypothesis that inflammation that results in neutrophil activation and release of histone/DNA complexes may trigger TTP. As shown, a1 $3^{-1}$ zebrafish exhibit increased levels of plasma VWF antigen, multimer size, and ability of thrombocytes to adhere to a fibrillar collagen-coated surface under flow. The a13-1zebrafish also show an increased rate of occlusive thrombus formation in the caudal venules after $\mathrm{FeCl}_{3}$ injury. More interestingly, a1 ${ }^{-/}$zebrafish exhibit $\sim 30 \%$ reduction in the number of total, immature, and mature thrombocytes with increased fragmentation of erythrocytes. Administration of a lysine-rich histone results in more severe and persistent thrombocytopenia and a significantly increased mortality rate in $a 13^{-1-}$ zebrafish than in wildtype (wt) ones. However, both spontaneous and histone-induced TTP in $113^{-}$zebrafish are rescued by the deletion of $v w f$. These results demonstrate a potentially mechanistic link between inflammation and the onset of TTP in light of severe A13 deficiency; the novel zebrafish models of TTP may help accelerate our understanding of pathogenic mechanisms and the discoveries of novel therapeutics for TTP and perhaps other arterial thrombotic disorders.

\section{Introduction}

Thrombotic thrombocytopenic purpura (TTP), resulting from severe deficiency of the plasma metalloprotease ADAMTS13 (A13), is characterized by thrombocytopenia and microangiopathic hemolytic anemia with various degrees of organ dysfunction. ${ }^{1,2}$ Most patients have immune-mediated TTP, caused by an immunoglobulin $\mathrm{G}(\mathrm{IgG})$ autoantibody that inhibits plasma A13 activity; ${ }^{3.7}$ rarely, TTP may be caused by a hereditary mutation or mutations in $A 13,8,9$ resulting in defective secretion of A13 protein, known as congenital TTP.

A13 is primarily produced in hepatic stellate cells $\mathrm{s}^{10,11}$ and released into the blood stream where it cleaves endothelium-derived ultra-large (UL) von Willebrand factor (VWF). ${ }^{12}$ The proteolysis of ULVWF by A13 is crucial for hemostasis ${ }^{1,13}$ and inflammation. ${ }^{14,15}$ When the ability to cleave ULVWF is compromised, due to deficiency of plasma A13 activity, ULVWF multimers accumulate on the surface of endothelium or at the site of vascular injury, recruit platelets from circulation, and promote formation of occlusive thrombi in small arterioles and capillaries - a pathognomonic feature of TTP. ${ }^{12,16}$ A13 and VWF are highly conserved from zebrafish to mammals, ${ }^{17,18}$ suggesting the importance of the A13/VWF axis in biology.

In the past two decades, studies have shown that patients with severe deficiency of plasma A13 activity often do not develop an acute TTP episode until a stressful
Haematologica 2020

Volume 105(4):1107-1119

\section{Correspondence:}

X. LONG ZHENG

xzheng@uabmc.edu or

longzheng01@gmail.com

Received: September 11, 2019.

Accepted: November 21, 2019.

Pre-published: November 21, 2019.

doi:10.3324/haematol.2019.237396

Check the online version for the most updated information on this article, online supplements, and information on authorship \& disclosures: www.haematologica.org/content/105/4/1107

(C)2020 Ferrata Storti Foundation

Material published in Haematologica is covered by copyright. All rights are reserved to the Ferrata Storti Foundation. Use of published material is allowed under the following terms and conditions:

https://creativecommons.org/licenses/by-nc/4.0/legalcode. Copies of published material are allowed for personal or internal use. Sharing published material for non-commercial purposes is subject to the following conditions:

https://creativecommons. org//icenses/by-nc/4.0/legalcode, sect. 3. Reproducing and sharing published material for commercial purposes is not allowed without permission in writing from the publisher. 
event. $^{19,20} A 13^{-/}$mice rarely develop spontaneous TTP unless they are challenged by a bacterial shigatoxin ${ }^{21,22}$ or a large dose of recombinant VWF., ${ }^{23,24}$ Baboons with acquired A13 deficiency develop features of TTP, but not a fatal condition. ${ }^{25}$ These findings indicate that additional environmental or genetic factors may be necessary for triggering severe TTP on top of A13 deficiency.

The potential triggers may be infection, inflammation, and pregnancy, etc. ${ }^{26}$ Infections or inflammation, including systemic lupus erythematosus, may cause activation of neutrophils, resulting in cell death - a process termed NETosis. ${ }^{27}$ This may lead to release of neutrophil granular contents including human neutrophil peptides (HNP), ${ }^{28}$ myeloperoxidase, ${ }^{29}$ and histone-DNA complexes. ${ }^{27}$ The plasma levels of HNP1-3, ${ }^{30,31}$ histone-DNA complexes, ${ }^{29}$ and other inflammatory mediators are significantly increased in patients with acute immune-mediated TTP. HNP1-3 may be prothrombotic ${ }^{31,32}$ or anti-thrombotic, ${ }^{33}$ depending on the context and their redox status. Increased plasma levels of histone-DNA complexes correlate with low platelet counts and disease severity. ${ }^{29}$ However, no direct evidence is available to date to support a causative role for any of these inflammatory mediators in the pathogenesis of TTP.

To test a hypothesis that inflammatory mediators, such as histones, may play a crucial role in triggering TTP when there is a severe deficiency of A13, we generated several novel zebrafish lines with a null mutation in $a 13, v w f$, and both using CRISPR/Cas9. These novel models were then used to assess the role of a lysine-histone, which is known to activate endothelial exocytosis, in triggering acute TTP. Zebrafish have been extensively used for modeling human diseases,$^{34}$ including thrombosis and hemostasis. ${ }^{35-}$ ${ }_{37}$ Zebrafish provide advantages over other animal models in terms of speed, cost, and high-throughput capability, enabling rapid assessment of the role of various environmental and genetic factors in triggering TTP and identification of novel therapeutics for TTP and perhaps other arterial thrombotic or inflammatory disorders.

\section{Methods}

\section{Zebrafish}

Zebrafish (Danio rerio) were used according to the protocol approved by Institutional the Animal Care and Use Committee. The guide RNA (gRNA) was designed using the CRISPR design tool (http://crispr.mit.edu/). A 69-nt oligonucleotide, consisting of a T7 promoter, a target sequence, and a gRNA scaffold, was synthesized (ThermoFisher, Waltham, MA, USA). The gRNA was then generated using a Guide-it sgRNA transcription kit (TakaraClontech, Mountain View, CA, USA). The Cas9 mRNA was synthesized from pT3TS-nCas9n using the mMESSAGE mMACHINE T3 kit (Life Technologies, Carlsbad, CA, USA). The final products $(2 \mathrm{~nL}$ with $12.5 \mathrm{pg} / \mathrm{nL}$ gRNA and $300 \mathrm{pg} / \mathrm{nL}$ Cas 9 mRNA) were co-injected into one-cell stage embryos of a double transgenic zebrafish (gata1-dsRed and fli1-eGFP). ${ }^{38,39}$

\section{Western blotting}

A capillary-based western blotting system (ProteinSimple, San Jose, CA, USA) was used to determine the presence of a13 protein in plasma and embryo lysate of zebrafish. The antibody was generated commercially (ABmart, Shanghai, China) by immunization of mice with nine synthetic peptides of zebrafish a13 protein (Online Supplementary Table S2).

\section{Agarose gel electrophoresis}

Plasma VWF multimers were determined by western blotting with anti-vwf IgG raised against zebrafish vwf peptides (ABclonal, Woburn, MA, USA) (Online Supplementary Figure S2) after electrophoresis on a $1.5 \%$ sodium dodecylsulfate-agarose gel. ${ }^{31}$

\section{Microfluidic assay}

Microchannels (Fluxion Bioscience, San Francisco, CA, USA) were coated with a fibrillar collagen $(100 \mu \mathrm{g} / \mathrm{mL})$. The surface was blocked with $0.5 \%$ bovine serum albumin. Pooled whole blood collected from ten adult zebrafish and anticoagulated with PPACK $(100 \mu \mathrm{M})$ was diluted with $50 \mu \mathrm{L}$ of phosphate-buffered saline (PBS) and perfused under 15 dyne $/ \mathrm{cm}^{2}$ over the collagen surface. The digital images were collected every 3 seconds for 120 seconds.

\section{Administration of a lysine-rich histone}

PBS or lysine-rich histone (H5505) (Sigma-Aldrich) $(200 \mathrm{mg} / \mathrm{kg}$ body weight) was injected intraperitoneally into adult zebrafish.

\section{Blood cell counts}

Zebrafish whole blood was diluted (1:51) with PBS (containing 4 mM EDTA). The total cell counts (per $\mu \mathrm{L}$ of blood) were determined using a Hemavet 950FS Hematology Analyzer (Drew Scientific, Miami Lakes, FL, USA). Additionally, flow cytometry (BD Biosciences, San Jose, CA, USA) was performed to differentiate erythrocytes, immature thrombocytes, and mature thrombocytes. The percentages of erythrocytes and thrombocytes were determined in 50,000 cells per sample. The number of thrombocytes per liter of whole blood was calculated using the formula: Thrombocyte count=Total cell counts per liter x Thrombocyte percentage $\mathrm{x}$ Dilution factor.

\section{Histological assessments}

Zebrafish were fixed in 4\% paraformaldehyde in PBS and embedded in paraffin for hematoxylin and eosin staining. Immunohistochemistry was then performed on the fixed tissue sections with a costumer-made rabbit anti-zebrafish vwf IgG (ABclonal, Woburn, MA, USA), followed by a horseradish peroxidase-conjugated anti-rabbit IgG and color reaction.

\section{Statistical analysis}

All data are presented as the mean \pm standard errors (SEM) or the median in a box-whisker plot unless specified in the figure legends. Kaplan-Meier survival analysis was performed with a logrank test. The Mann-Whitney test was used to determine the difference between two groups, while Krüskal-Wallis analysis was used to test the significance for more than three groups. All statistical analyses were carried out using Prism 7 software.

\section{Results}

\section{Generation and characterization of a13\% zebrafish}

To generate a stable $a 13^{-}$zebrafish line, an in vitro transcript consisting of gRNA and Cas 9 mRNA that targets a signal peptide of zebrafish a13 protein (Figure 1A) was injected into one-cell embryos of a double transgenic zebrafish (gata-1/dsRed and fli-1/eGFP) which expresses a red fluorescent protein under the gata-1 promoter in erythrocytes and immature thrombocytes and a green fluorescent protein under the fli-1 promoter in the entire vasculature and thrombocytes. Of 22 F0 founders randomly selected, 19 demonstrated the formation of heteroduplexes consistent with a heterozygous allele of a13 (Online Supplementary Figure 1A). The F0 founders were then out- 
bred with a wt zebrafish on the double transgenic background to generate F1, F2, and F3 progenies. Sanger sequencing confirmed the presence of various mutations, deletions, and insertions in the region encoding the signal peptide of a13 (Online Supplementary Figure S1B). The CRISPR design tool provided the sgRNA sequence that specifically targets the 913 gene. Any additional off-target was nearly eliminated after multiple generations of outbreeding with the wt zebrafish (Online Supplementary Figure S1C).

The resulting heterozygous siblings with an 8-bp deletion in a13 (Figure 1B) were selected for generating $a 13^{-1-}$ zebrafish for our subsequent studies. To facilitate rapid genotyping, we developed a PCR-melting curve strategy. The difference in the melting temperature of amplicons for the area of interest was dramatic between zebrafish with wt and those with a mutated gene (Online Supplementary Figure S2). This strategy provided rapid but reliable genotyping. The 8-nt deletion was predicted to introduce a premature stop within the signal peptide of a13, resulting in no expression of a13 protein, as demonstrated by an automated western blotting system (WES) in the embryo lysates (5 days post-fertilization) and plasma of all adult zebrafish tested (Figure 1C). Additionally,
A

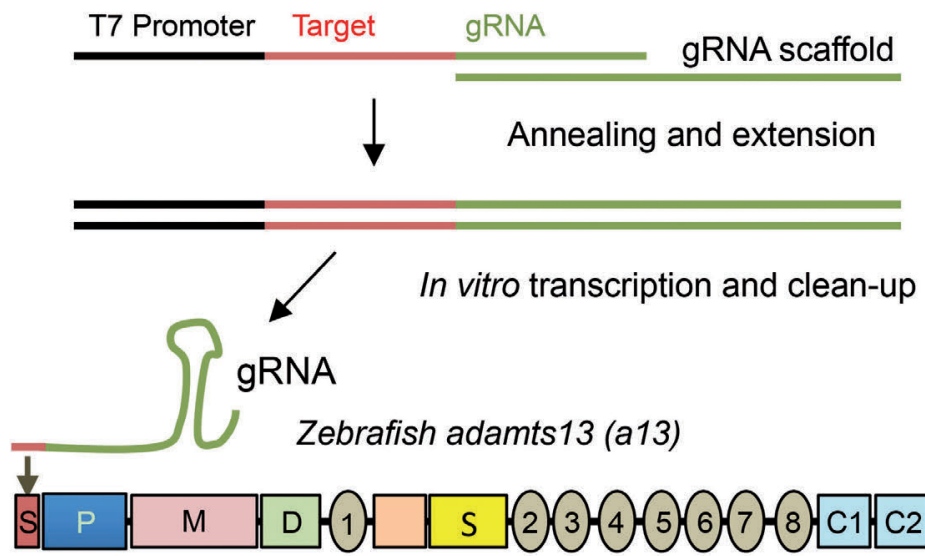

Figure 1. Generation and characterization of a13 zebrafish using CRISPR/Cas9. (A) An oligonucleotide consisting of a T7 promoter, a gene-specific target sequence (targeting the signal peptide coding region of zebrafish a13), and a guide RNA (gRNA) scaffold sequence was annealed and extended with the gRNA core sequence and then transcribed into a gRNA. (B) Sanger sequencing identified the wt (top) and 8-bp deletion (boxed) in a13 F2 progeny zebrafish (bottom, arrowhead). (C) Western blot by a capillary-based WES system demonstrates the absence $(a 13 \%$ ) and the presence (wt) of a13 protein $(\sim 220 \mathrm{kDa})$ in the lysate of zebrafish embryos ( 5 days post-fertilization) and in the plasma of 3-month old zebrafish, respectively. (D) A FRETS-VWF73 assay showed normal cleaving activity in the wt adult zebrafish plasma but not the a13\%. Such proteolytic activity was abrogated by the addition of 10 mmol/L EDTA. Pooled normal human plasma (NHP) was used as a positive control. Data represent the means \pm standard error of mean from three independent experiments.

B
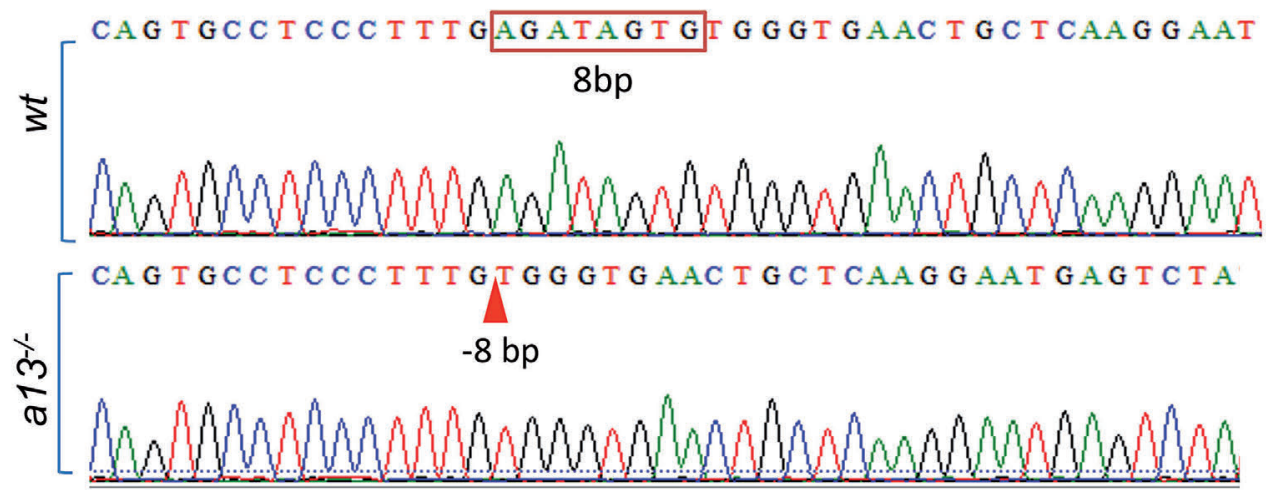

$$
\begin{array}{lll}
\text { GCCTCCCTTTGAGATAGTGTGGGTGAACTGCTCAAGGAATGAGTCT } & w t \\
\text { GCCTCCCTTTG - - - - - TGGGTGAACTGCTCAAGGAATGAGTCT } & a 13^{\%}(-8 \mathrm{bp})
\end{array}
$$

C



D

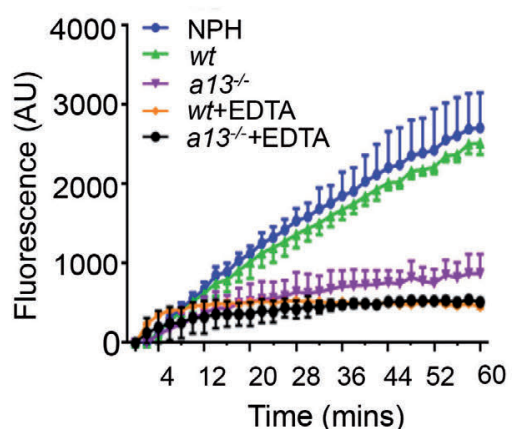


we demonstrated that there was no detectable a13 activity towards a FRETS-VWF73 substrate in zebrafish plasma (Figure 1D). Together, our results demonstrate that the a13 gene in zebrafish is successfully deleted.

\section{a13\% zebrafish are in a prothrombotic state}

Under unprovoked conditions, plasma levels of VWF antigen and multimer size were increased in $a 13^{-1}$ zebrafish compared with those in the wt controls (Figure $2 \mathrm{~A}-\mathrm{C}$ ). When a caudal vein of a zebrafish larva (gata$1 / \mathrm{dsRed}$ and $\mathrm{fli}-1 / \mathrm{eGFP})$ was exposed to $0.3 \% \mathrm{FeCl}_{3}$ (Figure 2D), an oxidative injury to vascular endothelium (the loss of green fluorescence) and an accumulation of erythrocytes (red fluorescence) were observed in $913^{-1}$ zebrafish (Figure 2E). The time to complete occlusion of the injured venule in $a 13^{-1}$ zebrafish was significantly shorter (mean \pm SEM, $2.1 \pm 0.3 \mathrm{~min}$ ) than that in the $w t$ controls $(13.6 \pm 1.8 \mathrm{~min})$ (Figure $2 \mathrm{~F})(P<0.001)$.

To confirm the incorporation of thrombocytes into the growing thrombus, we performed a similar experiment in a different transgenic zebrafish line, in which gata1/dsRed and cd41/eGFP were expressed to label erythrocytes (red) and thrombocytes (green), respectively. Confocal image analysis demonstrated the incorporation of thrombocytes and erythrocytes into the occlusive thrombus in the caudal vein of zebrafish larva after the oxidative injury (Figure 2G). Additionally, when anticoagulated whole blood was perfused over a fibrillar collagencoated surface under arterial shear $\left(15 \mathrm{dyne} / \mathrm{cm}^{2}\right)$, the surface coverage of $a 13^{-\%}$ thrombocytes was dramatically increased compared with that of wt thrombocytes (Figure $2 \mathrm{H})$ despite the similar initial rates of thrombocyte accumulation (Figure 2I). Supporting this, the length of thrombocyte-decorated VWF strings (mean \pm SEM) following perfusion of whole blood of $a 13^{-/-}$zebrafish $(87.0 \pm 1.1 \mu \mathrm{m})$ was significantly longer than that following perfusion of whole blood of wt zebrafish $(49.5 \pm 1.2 \mu \mathrm{m}) \quad(P<0.01)$ (Figure 2J). Together, these results demonstrate functional conservation of the a13/vwf axis in zebrafish hemostasis; and that the deletion of $a 13$ in zebrafish results in a prothrombotic phenotype.

\section{a13\% zebrafish develop a spontaneous but mild feature of thrombotic thrombocytopenic purpura}

Using total cell counts coupled with flow cytometry, we were able to quantify total, immature, and mature thrombocytes in whole blood accurately. Under fluorescent microscope, rare mature (green) and immature (orange) thrombocytes on the background of a large number of erythrocytes could be identified (Figure 3A), as previously described. ${ }^{39}$ The mean blood cell counts in $w t$ and $a 13^{-1}$ adult zebrafish were $3.2 \times 10^{12} / \mathrm{L}$ and $3.0 \times 10^{12} / \mathrm{L}$, respectively $(P>0.05)$. Total thrombocytes (mean \pm SEM) in $w t$ zebrafish account for $\sim 1 \%$ of total blood cells (i.e., $\left.33.9 \pm 1.2 \times 10^{9} / \mathrm{L}\right)$ while total thrombocytes in $a 13^{-1}$ zebrafish account for $\sim 0.7 \%$ of total blood cells (i.e. $\left.22.4 \pm 0.8 \times 10^{9} / \mathrm{L}\right)(P<0.0001)$ (Figure $\left.3 \mathrm{~B}\right)$. There was a similar degree of reduction $(\sim 30 \%)$ in both immature (mean \pm SEM, $16.5 \pm 0.6 \times 10^{9} / \mathrm{L}$ ) (Figure $3 \mathrm{C}$ ) and mature $(5.9 \pm 1.7$ $\mathrm{x} 10^{9} / \mathrm{L}$ ) (Figure 3D) thrombocytes in the $a 13^{-/}$group compared with those in the wt controls $\left(24.3 \pm 1.1 \times 10^{\circ} / \mathrm{L}\right.$ and $8.5 \pm 0.4 \times 10^{9} / \mathrm{L}$, respectively) $(P<0.0001)$. The ratio of immature to mature thrombocytes $(\sim 74: 26)$ was similar in both the $a 13^{-/}$and wt groups, suggesting no thrombocyte maturation defect in $a 13^{-}$zebrafish. There was no differ- ence in the number of total, immature, and mature thrombocytes observed between $w t$ and $a 13^{+/}$zebrafish (data not shown). The percentages of thrombocytes were also similar in different transgenic zebrafish lines (gata1-dsRed/fli1-eGFP vs. cd41-mCherry) (Online Supplementary Figure S3), which further confirmed the accuracy of thrombocyte counts based on eGFP/dsRed expression under the fli1/gata-1 promotor.

Microscopic examination of the peripheral blood smears revealed the presence of fragmented erythrocytes in $913^{-1}$ (Figure 3E, right), but not in wt (Figure 3E, left) and $a 13^{+/}$zebrafish (not shown). Quantitative analysis of more than ten blood smears from each group demonstrated a significant increase in the number of fragmented erythrocytes per high power field in $113^{-}$zebrafish (mean \pm SEM, $3.9 \pm 2.3)$ compared to $w t$ controls $(0.4 \pm 0.5) \quad(P<0.005)$ (Figure $3 \mathrm{~F})$. Together, our results demonstrate for the first time that $a 13^{-1}$ zebrafish could develop a spontaneous but mild phenotype of TTP.

\section{Lysine-rich histone induces a more severe and persistent thrombotic thrombocytopenic purpura phenotype in a13\% zebrafish than in wt ones}

The plasma levels of histone/DNA complexes are significantly elevated in patients with acute thrombotic microangiopathy, including immune-mediated TTP. ${ }^{29,40}$ When zebrafish were challenged with a single dose of lysine-rich histone, a significant drop in the number of total, immature, and mature thrombocytes within 24 to 48 $h$ was observed in both $w t$ and $a 13^{-/}$zebrafish. As shown, in wt zebrafish the number of total (Figure 4A) and immature thrombocytes (Figure 4B) recovered within $48 \mathrm{~h}$, while mature thrombocyte counts recovered $72 \mathrm{~h}$ after the histone challenge (Figure 4C). In $a 13^{-1}$ zebrafish, however, thrombocytopenia persisted after the histone challenge for the entire duration of observation (14 days) (Figure 4D). This was not the result of a decrease in immature thrombocytes (Figure 4E) but rather a reduction in mature thrombocytes (Figure 4F). At the end of 14 days after histone challenge, there were statistically significant differences in total (Figure 4G), immature (Figure 4H), and mature (Figure 4I) thrombocyte counts between wt and a13\% zebrafish. Histone challenge also resulted in a marked reduction of erythrocyte counts in $a 13^{-1 /}$ zebrafish but not wt ones (Online Supplementary Figure S4). Together, these results indicate that an inflammatory mediator such as histone may trigger acute TTP in individuals with severe A13 deficiency.

Kaplan-Meier survival analysis demonstrated a higher mortality rate $(\sim 30 \%)$ in $a 13^{-\%}$ zebrafish than in $w t$ zebrafish $(\sim 10 \%)$ following the histone challenge $(P=0.0002)$ (Figure 5A). No spontaneous death occurred in either $w t$ or $a 13^{-1}$ zebrafish over a 6-month period of observation without additional stress. To determine whether neutrophil activation and death or other tissue injuries occurred in zebrafish that were challenged with histones, we determined plasma levels of histone/DNA complexes in zebrafish prior to (D0) and 7 days (D7) after histone challenge which allowed for the exogenous histone to be cleared. We showed that while there was no statistically significant difference in the plasma levels of histone/DNA complexes between $w t$ and $a 13^{-/}$zebrafish prior to histone challenge, there were significantly higher levels of these complexes in $913^{-/}$than wt zebrafish 7 days after histone challenge when thrombocytopenia persisted 
A

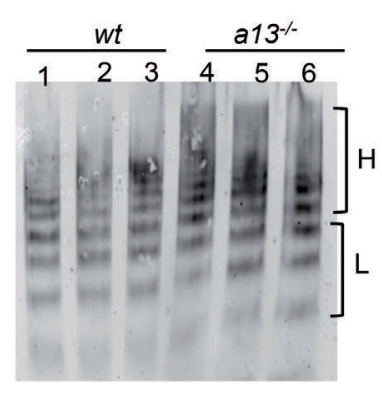

B


E

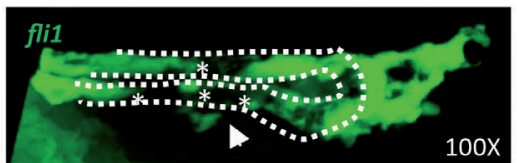

F
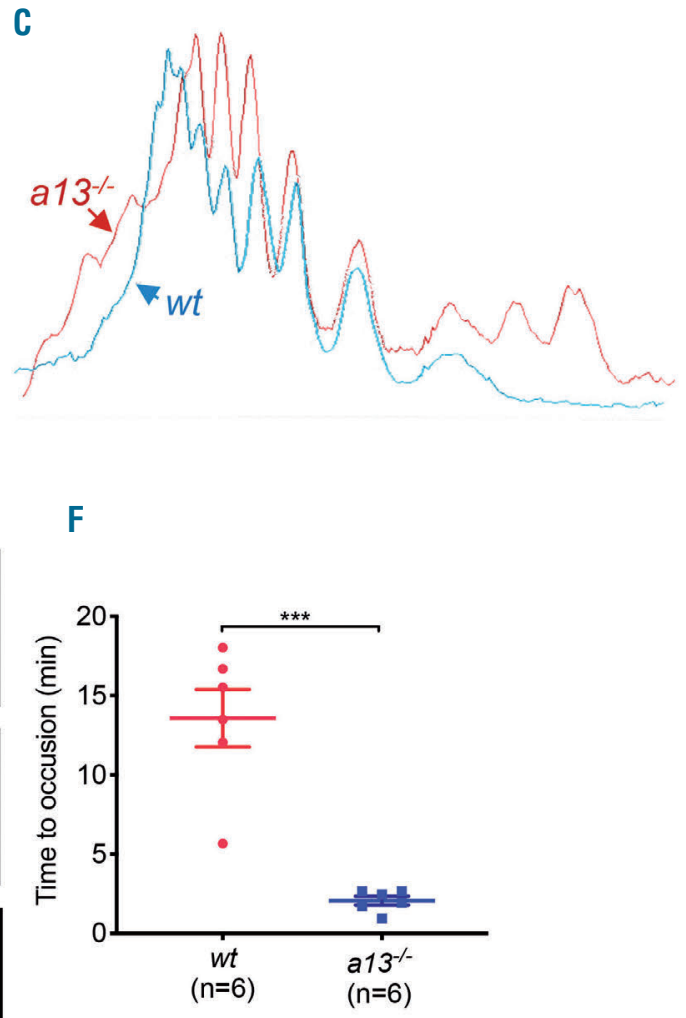

G
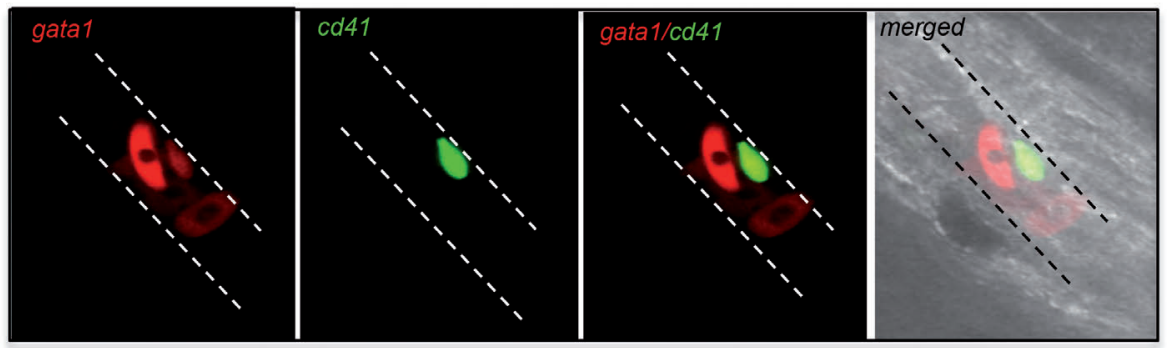

H

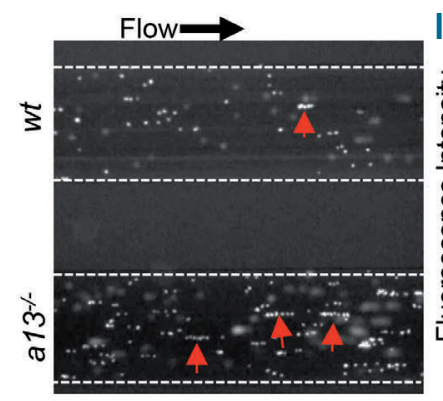

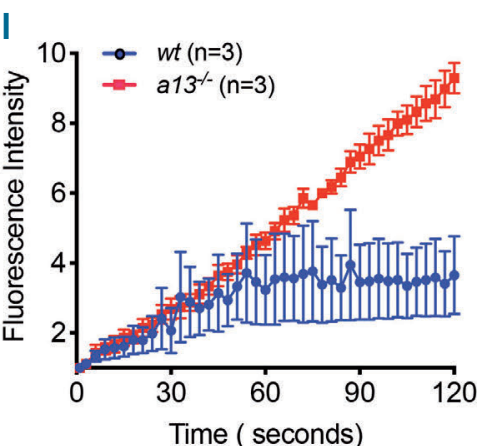

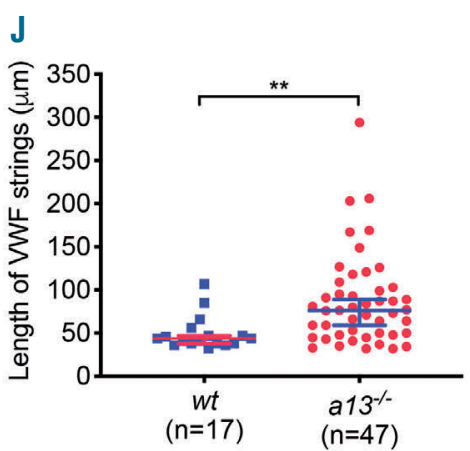

Figure 2. von Willebrand factor multimer and thrombus formation in wt and a13\% zebrafish. (A) Plasma von Willebrand factor (VWF) multimers in wt (lanes 1-3) and $a 13 \%$ (lanes 4-6) zebrafish aged 3-4 months. Here, $\mathrm{H}$ and $\mathrm{L}$ on the right margin indicate high and low molecular weight VWF multimers, respectively. (B) Quantification of plasma VWF antigen levels by densitometry (all multimers in each sample were scanned together) in wt and a13\% zebrafish. **P<0.01, MannWhitney test. (C) Representative densitometric scanning profiles of plasma vwf multimers in a13\% (red) and wt (blue) zebrafish. (D) The caudal areas of zebrafish endogenously expressing fli-1/eGFP and gata-1/sRed: the square or ovals show where a drop of $0.3 \%$ ferric chloride was placed topically. (E) Confocal images demonstrated the presence of thrombocytes (red) and loss of endothelial integrity (green) in the caudal vessels (outlined with dotted lines). The red arrows indicate the direction of blood flow from artery to vein. (F) Quantitative data showing the time to complete occlusion in the caudal vessel in wt and a13\% zebrafish after injury induced by $0.3 \%$ ferric chloride. $* * * P<0.005$, Mann-Whitney test. (G) Confocal image demonstrated the incorporation of both erythrocytes (red) and thrombocytes (green) into the occlusive thrombus in the caudal vein of wt zebrafish endogenously expressing gata-1/dsRed (red blood cells) and cd41/eGFP (thrombocytes) after being exposed to $0.3 \%$ ferric chloride. $(\mathrm{H})$ The surface coverage of fluorescent thrombocytes on a fibrillar collagen-coated surface in the microfluidic channel after perfusion of diluted whole blood (1:20), obtained from wt (top) and a13\% (bottom) zebrafish, under arterial shear (15 dyne/cm²). Red arrows indicate the thrombocyte-decorated strings formed on a vwf/collagen fiber. (I, J) The rate of fluorescence accumulation (means \pm SEM, $n=3$ ) (I) and the lengths of thrombocyte-decorated strings (means $\pm \mathrm{SEM})(\mathrm{J})$, following perfusion of a diluted whole blood from wt and $a 13 \%$ zebrafish. ** $P<0.01$, Mann-Whitney test. 
(Figure 5B). These results suggest that histone and/or histone/DNA complexes may not only be biomarkers for acute TTP, but also potential triggers for such a potentially fatal syndrome.

Histology of tissue sections revealed the presence of multiple large microvascular thrombi primarily in the liver (Figure 5C-i) and mesenteric vessels (not shown) in $913^{-1}$ zebrafish after histone challenge, but rare, small thrombi in wt zebrafish following the same treatment (Figure 5Cii). Under unprovoked conditions, there were occasional small microvascular thrombi in $a 13^{-/}$zebrafish (Figure 5Ciii), but not in wt zebrafish (Figure 5C-iv). Quantitation of microvascular thrombi in multiple stained tissue sections of various organs demonstrated that a single dose of his- tone resulted in the formation of significantly more microvascular thrombi in the $113^{-\%}$ than in the wt zebrafish $(P<0.0001)$ (Figure 5D. Immunohistochemical analysis showed the presence of VWF-rich thrombi in the liver parenchyma in $a 13^{-/}$zebrafish following histone challenge (Figure 5E, top). An omission of the primary antibody resulted in the complete absence of staining (Figure 5E, bottom). Agarose gel electrophoresis plus western blotting demonstrated the increased levels of plasma VWF antigen and multimer size $24 \mathrm{~h}$ (D1) after histone challenge in 913

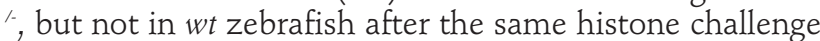
(Figure 5F). Also, consumption of ULVWF in zebrafish with acute TTP was observed between days 3-7 (Figure $5 \mathrm{~F})$, consistent with findings in patients. ${ }^{41}$ Together, these
A
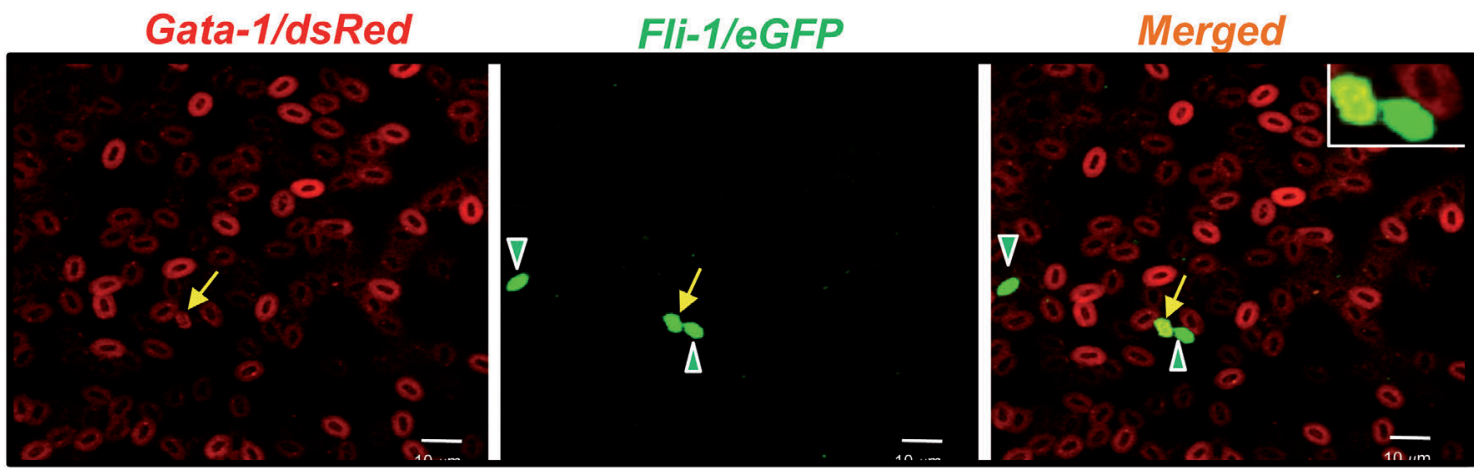

B

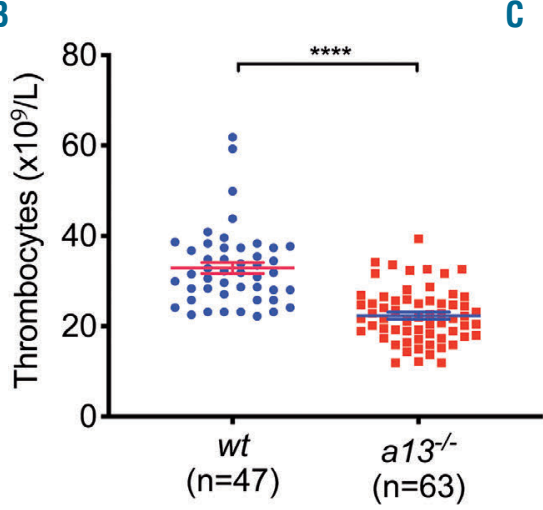

C



D



E

F


Figure 3. Microscopic and flow cytometric analyses of erythrocytes and/or thrombocytes in wt and a13\% zebrafish. (A) The fluorescent microscopic images of erythrocytes (left, red) on the background, rare mature thrombocytes (middle, green with open arrowheads) and immature thrombocytes (right, yellow with an arrow). (BD) The numbers of total (B), immature (C), and mature (D) thrombocytes in wt and a13\% zebrafish at the age of 3 months. Bars within the dots indicate the means \pm standard error of mean (SEM). $* * * * P<0.0001$, Mann-Whitney test. (E) Microscopic images of normal and fragmented nucleated erythrocytes (inset, arrows) in Geimsa-stained peripheral blood smears from wt and a13\% zebrafish as indicated. (F) The number of fragmented erythrocytes per high power field (HPF) in the peripheral blood smear of $w t$ and a13 zebrafish (100x). The lines within the individual dots represent the mean \pm SEM $(n=10)$. ***P<0.005, Mann-Whitney test. 
results demonstrate that a lysine-rich histone may trigger an acute episode of TTP in $a 13^{-/}$zebrafish.

\section{Generation and characterization of $v w f^{/ \bullet}$ and a13\%vwf/ zebrafish}

Lysine-rich histone is known to trigger the release of VWF from endothelial cells and enhance thrombus formation after vascular injury. ${ }^{42}$ To assess the contribution of VWF in this model, we first treated cultured endothelial cells with a lysine-histone, at the concentration used in zebrafish experiments, for various times. We found that lysine-histone induced rapid release of VWF from the cultured endothelial cells, which bound fluorescein-labeled platelets in whole blood from $a 13^{-}$mice under arterial flow (Online Supplementary Figure S5). We then deleted vwf from zebrafish to see if $v w f^{\prime}$ zebrafish were protected from the development of TTP, either spontaneously or triggered by histone. To this end, we generated a $v w^{\prime \prime}$ zebrafish line by targeting the $v w f$ gene encoding the propeptide region using the CRISPR-cas9 (Figure 6A). A 7 bp insertion mutant of $v w f$ was identified by Sanger sequencing (Figure 6B), which was predicted to form a
A



D

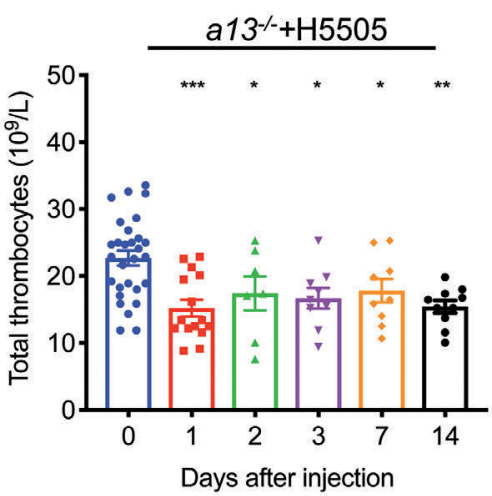

G

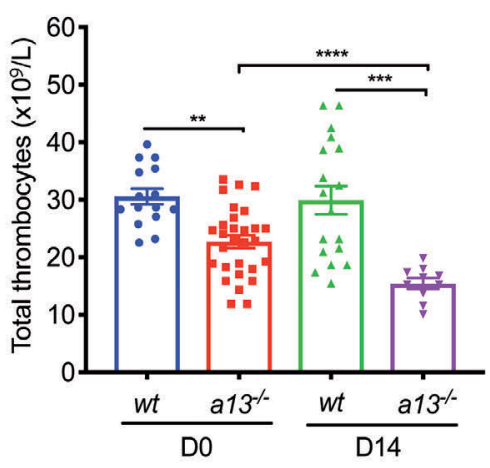

B

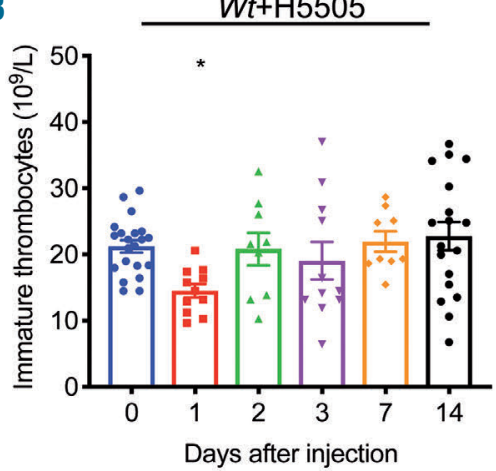

$E$



H



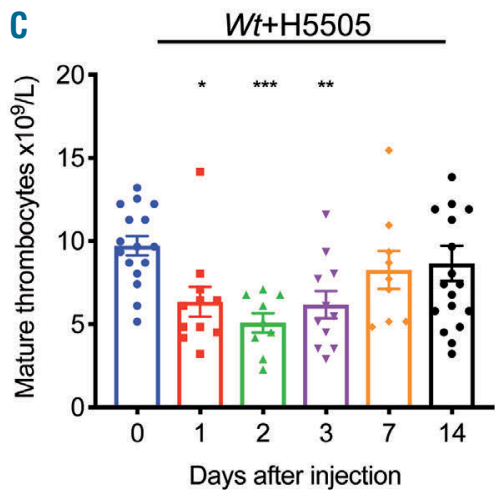

$\mathbf{F}$



I



Figure 4. Lysine-rich histone induced more severe and persistent thrombocytopenia in a13\% than in wt zebrafish. (A-C) The numbers of total (A), immature (B), and mature (C) thrombocytes per liter in wt zebrafish over 14 days after being challenged with $200 \mathrm{mg} / \mathrm{kg}$ body weight of a lysine-rich histone (H5505). (D-F) The numbers of total (D), immature (E), and mature (F) thrombocytes per liter in a13\% zebrafish over 14 days following the same dose of lysine-histone (H5505) challenge. (G-I) Direct comparisons of total $(\mathrm{G})$, immature $(\mathrm{H})$, and mature $(\mathrm{I})$ thrombocytes per liter between wt and a13-/- zebrafish prior to (D0) and 14 days (D14) following the histone (H5505) challenge. Kruskal-Wallis analysis was used to determine the difference among three or more groups (A-F), while the Mann-Whitney test was used to determine the difference between each two groups (G-I). All data are presented as dots, means, and \pm standard error of mean. $* P<0.05$, $* * P<0.01$, $* * * P<0.005$, and $* * * * P<0.0001$. 
premature stop within the propeptide, resulting in a vwfnull phenotype. Western blotting demonstrated the presence of vwf multimers in plasma of $w t$, but not $v w f^{\prime-}$ (Figure 6C) or $a 13^{-1} v w f^{-}$(not shown) zebrafish. These results demonstrate the successful deletion of the $v w f$ gene in zebrafish.

To further assess zebrafish $v w f$ function, we performed a microfluidic assay using whole blood from wt and $v w f^{\prime-}$ zebrafish. When perfused through a fibrillar collagen-coated surface under arterial shear, the final surface coverage of fluorescein-labeled thrombocytes (Figure 6E) and the rate of thrombocyte adhesion and aggregation (Figure 6F) onto the collagen surface were dramatically reduced in $v w f^{\prime}$ and $a 13^{-v w} f^{\prime}$ zebrafish compared with the wt controls $(P<0.0001)$. Moreover, the time to form an occlusive thrombus, determined by intravital microscopy, in the caudal vein in $v w f^{\prime}$ or $a 13^{-/} v w f^{\prime}$ zebrafish after $\mathrm{FeCl}_{3}$ injury was significantly prolonged when compared to that in the $w t$ controls (Figure 6D). All wt zebrafish formed occlusive thrombi within 20 min after $\mathrm{FeCl}_{3}$ injury, but nearly $50 \%$ of $v w f^{\wedge}$ or $a 13^{-v w f^{\wedge}}$ zebrafish failed to form occlusive thrombi. These results demonstrate the relatively deficient hemostatic functions in $v w f^{\prime}$ and $a 13^{-/} v w f^{\prime}$ zebrafish.

$v w f /$ and $a 13 \% v w f /{ }^{\prime}$ zebrafish are protected from developing spontaneous and histone-induced thrombocytopenia

Under unprovoked conditions, $v w f^{\wedge}$ zebrafish with or without circulating plasma a13 exhibited normal counts of total (Figure 7A), immature (Figure 7B), and mature (Figure 7C) thrombocytes. Moreover, when challenged with a lysine-rich histone (H5505), vwf or a13 ${ }^{-1} v w f^{\prime}$ zebrafish exhibited no significant reduction in the numbers of total (Figure 7D, G), immature (Figure 7E, H), and mature (Figure 7F, I) thrombocytes over a 7-day period. However, 7 days after the histone challenge, there were significantly lower numbers of total (Figure 7J), immature (Figure 7K), and mature (Figure 7L) thrombocytes in $a 13^{-1-}$ than in $a 13^{-1} v w f^{/}$zebrafish. Together, these results demonstrate that $v w f^{\prime}$ zebrafish with or without circulating A13 are protected from developing spontaneous or histone-induced TTP.
A

$$
\begin{aligned}
& \text {... } w t / P B S+w t / \mathrm{H} 5505 \\
& \text { - } a 13^{-/} / \mathrm{PBS}+a 13^{-/ / H 5505}
\end{aligned}
$$

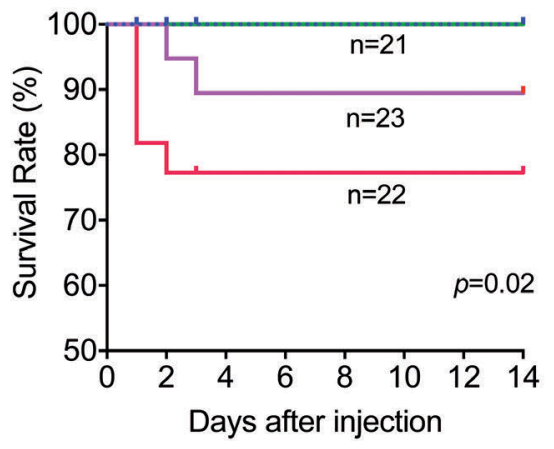

D

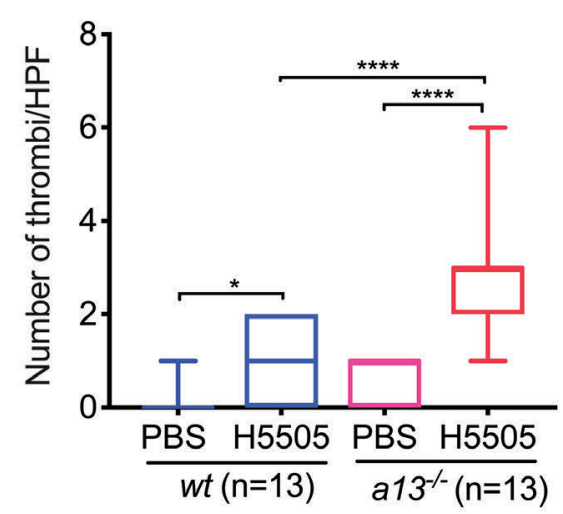

B

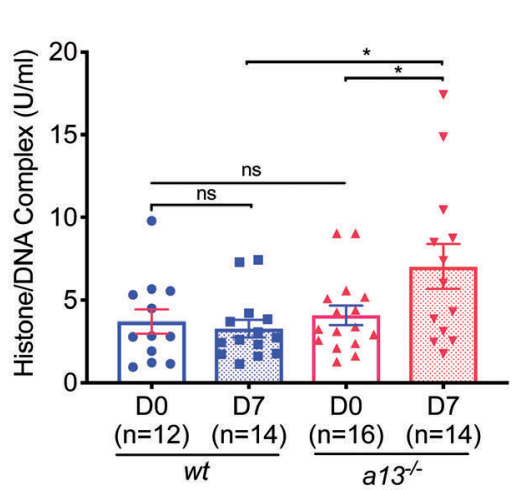

E



C

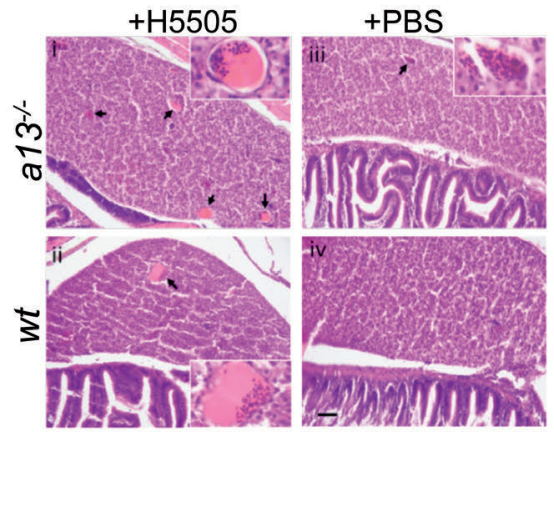

F



Figure 5. Kaplan-Meier survival analysis, plasma histone/DNA complexes, histology and von Willebrand factor multimers in wt and a13 zebrafish after histone challenge. (A) Survival rate (\%) over a 2-week period in wt and a13 zebrafish after being challenged with phosphate-buffered saline (PBS) or lysine-rich histone (H5505) (200 mg/kg body weight). (B) Plasma levels of histone-DNA complexes in wt and a13\% zebrafish prior to (D0) and day 7 (D7) following the histone (H5505) challenge. (C) Histological images of occlusive microvascular thrombi (arrowheads) in the liver tissue from a13\% (top) and wt (bottom) zebrafish following lysine-histone (H5505) (left) or PBS challenge (right). (D) The numbers of microvascular thrombi per tissue section (box-and-whisker plot, with minimum to maximum) in wt and $13 \%$ zebrafish following the histone (H5505) or PBS challenge. A Mann-Whitney test was performed to test differences between two groups. $* P<0.05$ $* * P<0.01, * * * P<0.005$, and $* * * * P<0.001$. (E) Immunohistochemical staining showing von Willebrand factor (VWF)-rich microvascular thrombi (top, arrowheads). Scale bar represents $200 \mu \mathrm{m}$ in length. (F) Plasma vwf multimer distribution in wt and a13\% zebrafish prior to (D0), 1, 3, and 7 days after the histone (H5505) challenge. Here, $\mathrm{H}$ and $\mathrm{L}$ on the right margin indicate high and low molecular weight VWF multimers, respectively. 


\section{Discussion}

The present study represents the first establishment and characterization of zebrafish models of TTP and the illustration of a potential trigger for the disorder on top of A13 deficiency. The a13 zebrafish exhibit a prothrombotic phenotype with increased levels of plasma VWF antigen and multimer size. This results in an enhanced ability of thrombocytes to adhere and aggregate on a fibrillar collagen surface under arterial flow. Additionally, a13zebrafish show an accelerated rate of thrombus formation following oxidative injury. Such a prothrombotic phenotype in $a 13^{-}$zebrafish is completely rescued when $v w f$ is genetically deleted, suggesting that the prothrombotic phenotype in a13 zebrafish can be attributed to the enhanced VWF function in vivo.

More interestingly, a13\% zebrafish develop a spontaneous but mild TTP phenotype, which is characterized by low thrombocyte and erythrocyte counts with an increased number of fragmented red blood cells, as well as the formation of small occlusive thrombi in organ tissues. Such a TTP phenotype in the $113^{-1}$ zebrafish is significantly worsened and sometimes fatal following challenge with a lysine-rich histone, which has previously been shown to trigger endothelial exocytosis and release of ULVWF multimers from Weibel-Palade bodies of activated endothelium. ${ }^{42}$ An intravenous administration of histones induces transient thrombocytopenia in mice, ${ }^{43}$ although the mechanism underlying this transient effect is not known. We show here that both the spontaneous and histone-induced

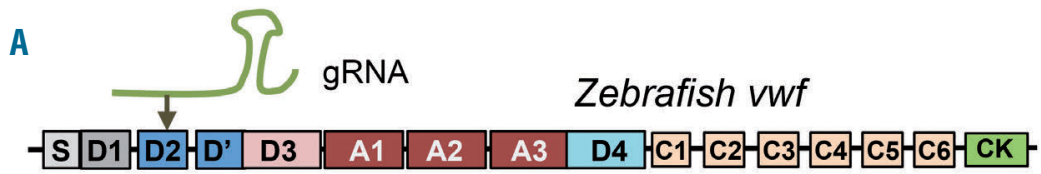

\section{AGATTGCAGGCAGCGAAGCTTCTCCATACTGGGTAAAACA AGATTGCAGGCAGCGAAGCTTCTCCATgGGTAAaACTGGGTAAAACA $\quad v w f^{\prime-}(+7 \mathrm{bp})$}

B
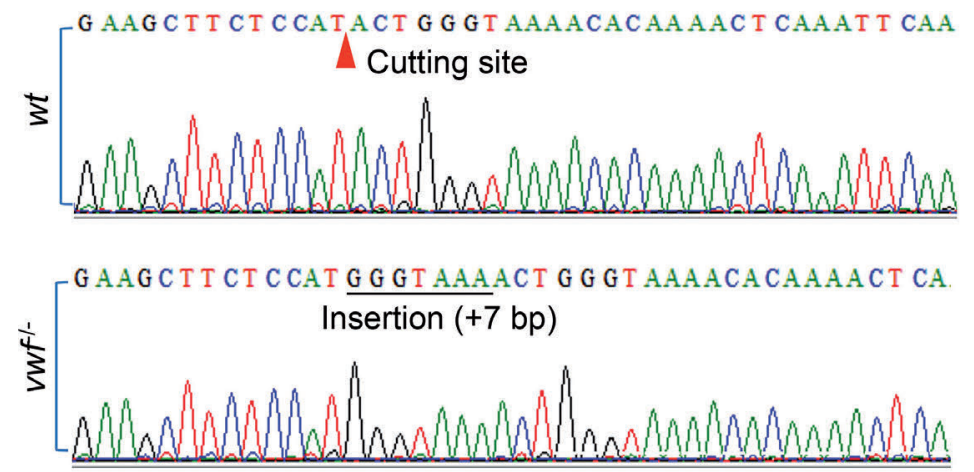

C

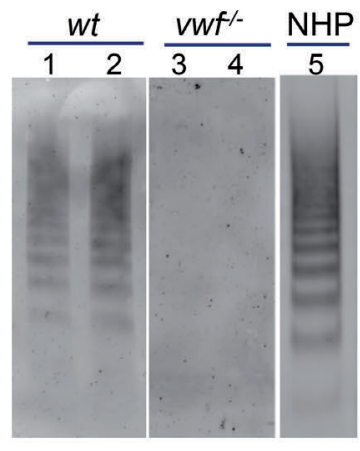

D



E



$\mathrm{F}$



Figure 6. Generation and characterization of $v w f /$ and a13 vwf/ zebrafish using CRISPR/Cas9. (A) A guide RNA (gRNA) oligonucleotide was used to target the propeptide D2 domain-coding region of zebrafish vwf. (B) Sanger sequencing confirming the wildtype (wt) sequence and the 7-bp insertion in the targeted region, as indicated, which results in vwf null by causing a frameshift and introduction of a premature stop. (C) Agarose gel electrophoresis and western blotting with a specific antibody raised against zebrafish VWF, demonstrating the presence of VWF multimers in wt plasma ( 2 and $4 \mu \mathrm{L}$ in lane 1 and 2 , respectively), but not in vwf plasma ( 2 and $4 \mu \mathrm{L}$ in lanes 3 and 4 ) at the age of 3 months. Normal human plasma (NHP) $(0.5 \mu \mathrm{L}$ ) was used as a positive control (lane 5$)$, run in a separate gel and blotted with an anti-human VWF IgG. (D) The time to form occlusive thrombi in wt, vwf/, and a13 ${ }^{\prime / v} \mathrm{wf}^{\prime}$ zebrafish after FeCl $\mathrm{Finj}_{3}$ in at the caudal venule (box-and-whisker plots, with minimum to maximum). ( $\mathrm{E}, \mathrm{F})$ The representative surface coverage and rate of accumulation of fluorescent thrombocytes (mean \pm standard error of mean, $\mathrm{n}=3$ ), respectively, on a fibrillar collagen surface following perfusion of a diluted whole blood from $w t, v w f^{\prime}$, and $a 13^{\prime} v^{\prime} f^{\prime} /$ zebrafish under arterial shear $\left(15 \mathrm{dyne} / \mathrm{cm}^{2}\right)$, as indicated. 
TTP phenotype in $a 13^{-\%}$ zebrafish is essentially eliminated when $v w f$ is genetically deleted. Consistent with this result, the deletion of $v w f$ also confers a $13^{-1}$ zebrafish embryos resistance to histone challenge. Together, these findings indicate that VWF contributes to the pathophysiology of spontaneous and histone-induced thrombocytopenia or TTP in $a 13^{-/}$zebrafish.
The structure and function of VWF are conserved from zebrafish to mammals with an overall similarity at the protein level of $\sim 45 \% .{ }^{17}$ Plasma VWF multimers in zebrafish, visualized for the first time in this study, and recombinantly expressed VWF multimers reported previously, ${ }^{17}$ are quite similar in pattern. Furthermore, zebrafish VWF is shown to interact with thrombocytes during
A



D

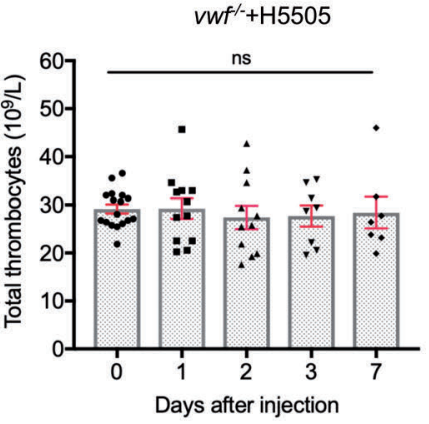

G

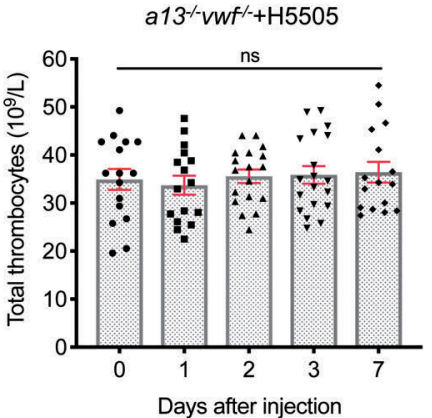

J



B



E

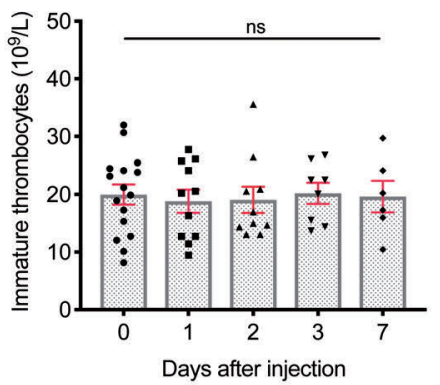

$\mathrm{H}$

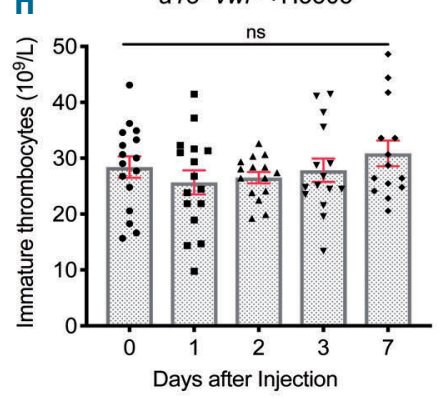

K

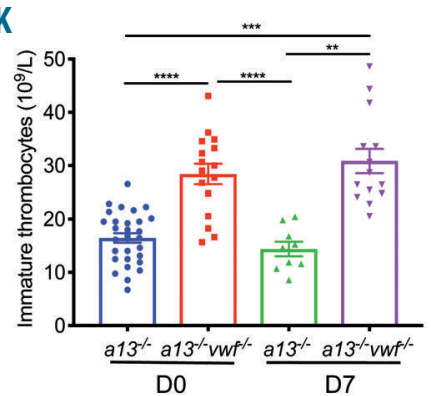

C



F
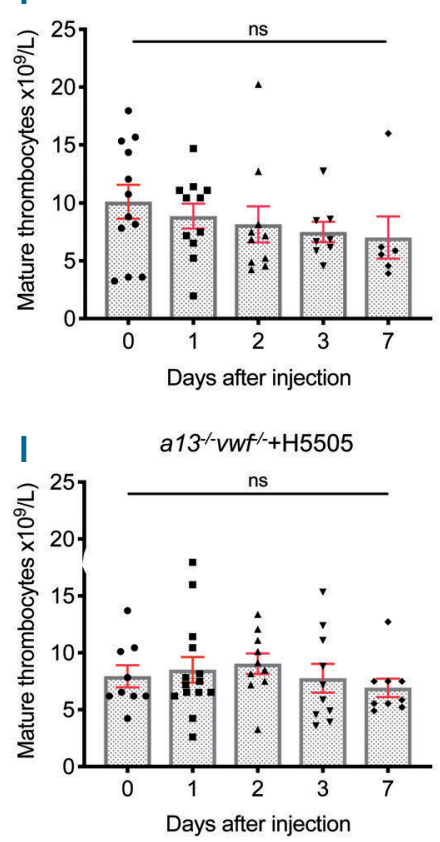

L

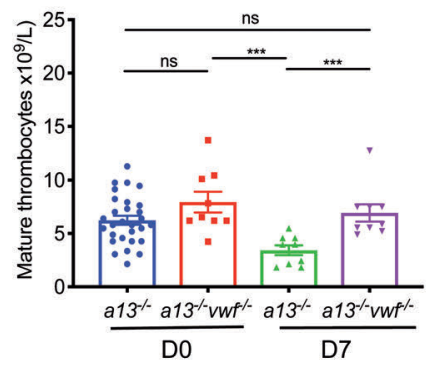

Figure 7. Deletion of vwf rescued histone-induced thrombotic thrombocytopenic purpura in a13\% zebrafish. (A-C) The number of total (A), immature (B), and mature (C) thrombocytes per liter in wt, vwf $\%$ a 13\%, and vwf/a13\% zebrafish at age of 3 months without any challenge. Kruskal-Wallis analysis was performed. (D-F) The number of total (D), immature (E), and mature (F) thrombocytes per liter in vwf/zebrafish prior to (DO), 1, 2, 3, and 7 days following histone (H5505) challenge. (GI) The number of total $(\mathrm{G})$, immature $(\mathrm{H})$, and mature (I) thrombocytes per liter in $a 13 / v w f^{\prime}$ zebrafish prior to (D0), 1, 2, 3, and 7 days following histone (H5505) challenge. (J-L) Direct comparisons of total (J), immature (K), and mature (L) thrombocytes per liter between a13 and a13 vwf zebrafish prior to (D0) and 7 days (D7) after histone (H5505) challenge. All data are expressed as individual values (dots) and the means \pm standard error of mean. Kruskal-Wallis analysis and the Mann-Whitney test were used to determine the statistical significances among three or more groups and between two groups, respectively. ns: no statistically significant difference; $* P<0.05, * * P<0.01, * * * P<0.005$, and $* * * * P<0.001$, respectively. 
thrombus formation in our study. In the absence of circulating VWF, thrombocytes fail to adhere to the fibrillar collagen surface under flow and thrombus formation is significantly impaired in the small vessels after injury. During the revision of this manuscript, similar hemostatic defects were reported to occur when $v w f$ is independently knocked out. ${ }^{44}$ These findings support the crucial physiological function of zebrafish VWF in mediating thrombocyte adhesion/aggregation and thrombus formation.

The A13 protein in zebrafish is also conserved with a similar domain structure to that in mammals except for a longer signal peptide and propeptide. There is $\sim 52 \%$ sequence similarity at the A13 protein level between zebrafish and humans (or mice). Surprisingly, zebrafish plasma A13 is able to cleave the human FRETS-rVWF73 ${ }^{45}$ at the similar rate as human plasma A13 does. This activity is abrogated by EDTA, which chelates divalent metal ions, suggesting that zebrafish A13 is also a metalloprotease. Additional evidence to support this notion is that plasma VWF multimers and thrombocyte-decorated VWF strings on the fibrillar collagen surface under flow are significantly increased in $a 13^{\%}$ zebrafish when compared with those in wt controls. The rate of thrombus formation in the caudal venules after oxidative injury is also dramatically accelerated in $a 13^{-}$zebrafish. More interestingly, a13 $3^{\%}$ zebrafish develop a spontaneous but non-fatal TTP phenotype, similar to that in some patients with congeni- tal $\operatorname{TTP}^{20,46}$ and in a baboon model of acquired TTP induced by passive immunization. ${ }^{25}$ Together, these results suggest that mild TTP may be compatible with life, but the affected individuals may have a significantly increased risk of developing catastrophic or potentially fatal TTP when an additional environmental trigger, such as infection or pregnancy, is present.

Infection or systemic inflammation often precedes the onset of TTP, which is thought to activate neutrophils, resulting in the release of their intracellular contents, including antimicrobial HNP1-3, proteolytic enzymes, nucleic acids, and histone-DNA complexes. ${ }^{29,42}$ Plasma levels of histone-DNA complexes are markedly elevated in patients with acute TTP. ${ }^{40}$ Although the causes of elevated levels of plasma histone-DNA are not fully understood. Histone-DNA has been shown to have deleterious effects with prothrombotic properties. Histones may activate platelets, enhance thrombin generation, ${ }^{47}$ induce exocytosis of endothelial Weibel-Palade bodies, and trigger the release of ULVWF from endothelium. ${ }^{42}$ Histones may also activate complement, resulting in the formation of a membrane attack complex that injures endothelium. ${ }^{48}$ Based on our results and those published in the literature, we hypothesize that histones, likely derived from NETosis and other cell death after injury, are potent triggers for TTP. When plasma A13 activity is present, the released ULVWF multimers are rapidly cleaved into smaller forms.

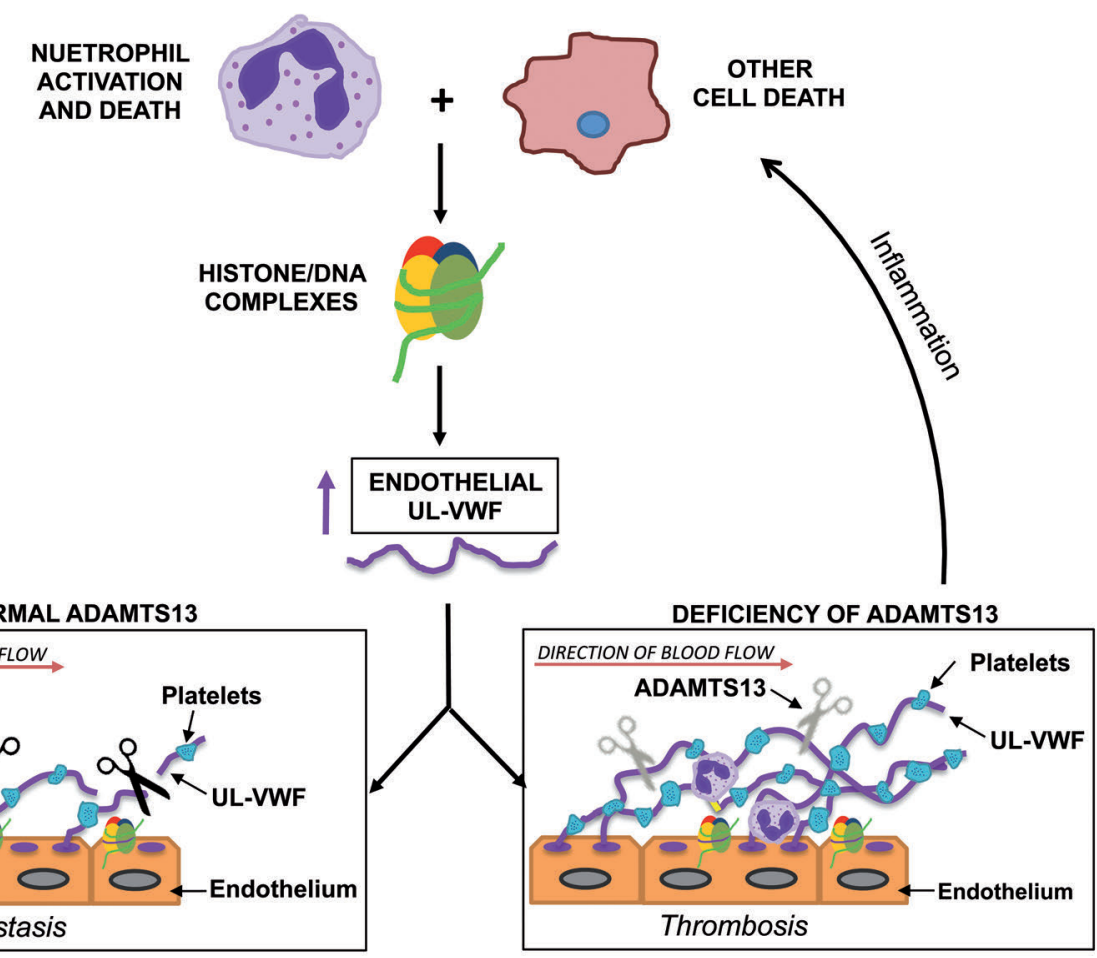

Figure 8. A proposed working model depicts how neutrophil activation and the release of histones induce microvascular thrombosis in a13\% zebrafish. Infection and/or systemic inflammation may activate neutrophils and result in neutrophil death and tissue injury, leading to the release of their cellular contents including histone/DNA complexes or free histone. Histone/DNA complexes or free histone may then bind to endothelial receptors, which triggers exocytosis of Weibel-Palade bodies and the release of ultralarge multimers of von Willebrand factor (UL-VWF) from activated endothelium. These hyperactive UL-VWF multimers are rapidly removed by plasma ADAMTS13, so that normal hemostasis is maintained. However, when plasma ADAMTS13 is absent, as in cases of hereditary or acquired thrombotic thrombocytopenic purpura (TTP), the UL-VWF strings are not removed from the endothelial surface, resulting in an accumulation of platelets/thrombocytes and neutrophils, leading to thrombus formation and acute inflammation at sites of vascular injury. In the end, activation of the coagulation cascade, complement, and neutrophils may occur, resulting in further tissue damage and microvascular thrombosis, the characteristic pathological feature of TTP. 
This prevents excessive thrombus formation at the sites of vascular injury; when plasma A13 activity is absent, the released ULVWF remain anchored on the endothelial surface, which can capture flowing platelets in circulation, resulting in occlusive thrombi and acute inflammation at the sites of vascular injury. This creates a positive feedback, resulting in more inflammation and cell death, which leads to the formation of a vicious cycle (Figure 8).

In conclusion, we report the generation and characterization of several novel zebrafish lines $\left(a 13^{-1}, v w f^{\prime}\right.$, and $\left.a 13^{-} v w f^{\wedge}\right)$ and zebrafish models of TTP. With these new tools, we are able to demonstrate potentially mechanistic links between infection, innate immunity (such as neutrophil activation and the release of histone/DNA complexes), and the onset and progression of TTP in the setting of severe deficiency of plasma A13 activity. These novel zebrafish models with a robust high-throughput screening capability could help accelerate the discovery of potentially novel therapeutics for TTP and many other arterial thrombotic disorders, in which treatment options are quite limited.

\section{Acknowledgments}

This study was supported in part by a grant from the National Institutes of Health (HL126724) to XLZ and a postdoctoral fellowship (18POST33960098) to LZ from the American Heart Association. The authors also thank Drs. Daniel Gorelick and Shannon Romano from the Department of Pharmacology and Toxicology at $U A B$ for providing a double transgenic zebrafish line expressing fli-1eGFP and gata-1dsRed, Dr. Susan Farmer at the Zebrafish Research Facility for providing wildtype zebrafish, and Drs. Robert I. Handin and Dongdong Ma, at Harvard Medical School, Boston, MA for helping us with the cd41-mCherry transgenic zebrafish line. The authors thank Nicole Kocher at UAB for her assistance and critical reading of this paper prior to its submission.

\section{References}

1. Zheng XL. ADAMTS13 and von Willebrand factor in thrombotic thrombocytopenic purpura Annu Rev Med. 2015;66:211-225.

2. Rock GA, Shumak KH, Buskard NA, et al. Comparison of plasma exchange with plasma infusion in the treatment of thrombotic thrombocytopenic purpura. Canadian Apheresis Study Group. N Engl J Med. 1991;325(6):393-397.

3. Tsai HM Lian EC Antibodies to von Willebrand factor-cleaving protease in acute thrombotic thrombocytopenic purpura. N Eng J Med. 1998;339(22):1585-1594.

4. Zheng XL, Wu HM, Shang D, et al. Multiple domains of ADAMTS13 are targeted by autoantibodies against ADAMTS13 in patients with acquired idiopathic thrombotic thrombocytopenic purpura. Haematologica. 2010;95(9):1555-1562.

5. Ostertag EM, Bdeir K, Kacir S, et al. ADAMTS13 autoantibodies cloned from patients with acquired thrombotic thrombocytopenic purpura: 2. Pathogenicity in an animal model. Transfusion. 2016;56(7): 1775-1785.

6. Casina VC, Hu W, Mao JH, et al. High-resolution epitope mapping by HX MS reveals the pathogenic mechanism and a possible therapy for autoimmune TTP syndrome. Proc Natl Acad Sci U S A. 2015;112(31): 9620-9625.

7. Thomas MR, de Groot R, Scully MA, Crawley JT. Pathogenicity of antiADAMTS13 autoantibodies in acquired thrombotic thrombocytopenic purpura. EBioMedicine. 2015;2(8):942-952

8. Levy GG, Nichols WC, Lian EC, et al. Mutations in a member of the ADAMTS gene family cause thrombotic thrombocytopenic purpura. Nature. 2001;413(6855): 488-494.

9. Zheng XL, Sadler JE. Pathogenesis of thrombotic microangiopathies. Annu Rev Pathol. 2008;3:249-277.

10. Uemura M, Tatsumi K, Matsumoto M, et al. Localization of ADAMTS13 to the stellate cells of human liver. Blood. 2005;106(3):922924.
11. Zhou W, Inada M, Lee TP, et al. ADAMTS13 is expressed in hepatic stellate cells. Lab Invest. 2005;85(6):780-788.

12. Dong JF, Moake JL, Nolasco L, et al. ADAMTS-13 rapidly cleaves newly secreted ultralarge von Willebrand factor multimers on the endothelial surface under flowing conditions. Blood. 2002;100(12):4033-4039.

13. Sadler JE. von Willebrand factor: two sides of a coin. J Thromb Haemost. 2005;3(8):1702-1709.

14. Chauhan AK, Kisucka J, Brill A, et al. ADAMTS13: a new link between thrombosis and inflammation. I Exp Med. 2008;205(9):2065-2074

15. De Meyer SF, Savchenko AS, Haas MS, et al. Protective anti-inflammatory effect of ADAMTS13 on myocardial ischemia/reperfusion injury in mice. Blood. 2012;120(26):5217-5223

16. Arya M, Anvari B, Romo GM, et al. Ultralarge multimers of von Willebrand factor form spontaneous high-strength bonds with the platelet glycoprotein Ib-IX complex: studies using optical tweezers. Blood. 2002;99(11):3971-3977.

17. Ghosh A, Vo A, Twiss BK, et al. Characterization of zebrafish von Willebrand factor reveals conservation of domain structure, multimerization, and intracellular storage. Adv Hematol. 2012;2012:214209.

18. Jin SY, Skipwith CG, Zheng XL. Amino acid residues $\operatorname{Arg}(659), \operatorname{Arg}(660)$, and $\operatorname{Tyr}(661)$ in the spacer domain of ADAMTS13 are critical for cleavage of von Willebrand factor. Blood. 2010;115(11):2300-2310.

19. Fujimura $Y$, Kokame $K$, Yagi $H$, et al. Hereditary deficiency of ADAMTS13 activity: Upshaw-Schulman syndrome. In: Rodgers GM, ed. ADAMTS13 Biology and Disease. Cham, Heidlberg, New York, Dordrecht, London: Springer. 2015:73-90.

20. Fujimura $Y$, Matsumoto M, Isonishi A, et al. Natural history of Upshaw-Schulman syndrome based on ADAMTS13 gene analysis in Japan. J Thromb Haemost. 2011;9 Suppl 1:283-301.

21. Motto DG, Chauhan AK, Zhu G, et al. Shigatoxin triggers thrombotic thrombocy- topenic purpura in genetically susceptible ADAMTS13-deficient mice. J Clin Invest. 2005;115(10):2752-2761.

22. Jin SY, Xiao J, Bao J, et al. AAV-mediated expression of an ADAMTS13 variant prevents shigatoxin-induced thrombotic thrombocytopenic purpura. Blood. 2013;121(19): 3825-3829.

23. Schiviz A, Wuersch K, Piskernik C, et al. A new mouse model mimicking thrombotic thrombocytopenic purpura: correction of symptoms by recombinant human ADAMTS13. Blood. 2012;119(25):6128 6135.

24. Pickens B, Mao Y, Li D, et al. Platelet-delivered ADAMTS13 inhibits arterial thrombosis and prevents thrombotic thrombocytopenic purpura in murine models. Blood. 2015;125(21):3326-3334.

25. Feys HB, Roodt I, Vandeputte N, et al Thrombotic thrombocytopenic purpura directly linked with ADAMTS13 inhibition in the baboon (Papio ursinus). Blood. 2010;116(12):2005-2010.

26. Furlan M, Lammle B. Aetiology and pathogenesis of thrombotic thrombocytopenic purpura and haemolytic uraemic syndrome: the role of von Willebrand factor-cleaving protease. Best Pract Res Clin Haematol. 2001;14(2):437-454

27. Martinod K, Wagner DD. Thrombosis: tangled up in NETs. Blood. 2014;123(18):27682776

28. Ganz T, Selsted ME, Szklarek D, et al Defensins. Natural peptide antibiotics of human neutrophils. J Clin Invest. 1985;76 (4):1427-1435.

29. Fuchs TA, Kremer Hovinga JA, Schatzberg D, Wagner DD, Lammle B. Circulating DNA and myeloperoxidase indicate disease activity in patients with thrombotic microangiopathies. Blood. 2012;120(6):1157-1164.

30. Cao W, Pham HP, Williams LA, et al. Human neutrophil peptides and complement factor $\mathrm{Bb}$ in pathogenesis of acquired thrombotic thrombocytopenic purpura. Haematologica. 2016;101(11):1319-1326.

31. Pillai VG, Bao J, Zander CB, et al. Human neutrophil peptides inhibit cleavage of von Willebrand factor by ADAMTS13: a poten- 
tial link of inflammation to TTP. Blood. 2016;128(1):110-119

32. Higazi AA, Ganz T, Kariko K, Cines DB. Defensin modulates tissue-type plasminogen activator and plasminogen binding to fibrin and endothelial cells. J Biol Chem. 1996:271(30):17650-17655.

33. McDaniel JK, Abdelgawwad MS, Hargett A, et al. Human neutrophil peptide- 1 inhibits thrombus formation under arterial flow via its terminal free cysteine thiols. J Thromb Haemost. 2019;17(4):596-606.

34. Dooley K, Zon LI. Zebrafish: a model system for the study of human disease. Curr Opin Genet Dev. 2000;10(3):252-256.

35. Jagadeeswaran P, Cooley BC, Gross PL, Mackman N. Animal models of thrombosis from zebrafish to nonhuman primates: use in the elucidation of new pathologic pathways and the development of antithrombotic drugs. Circ Res. 2016;118 (9):1363-1379.

36. Jagadeeswaran P. Zebrafish: a tool to study hemostasis and thrombosis. Curr Opin Hematol. 2005:12(2):149-152.

37. Weyand AC, ShavitJA. Zebrafish as a model system for the study of hemostasis and thrombosis. Curr Opin Hematol. 2014;21 (5):418-422.

38. Delov V, Muth-Kohne E, Schafers C, Fenske M. Transgenic fluorescent zebrafish $\operatorname{Tg}(f l i 1: E G F P) y(1)$ for the identification of vasotoxicity within the $z F E T$. Aquat Toxicol. 2014;150:189-200.

39. Jagadeeswaran P, Lin S, Weinstein B, Hutson A, Kim S. Loss of GATA1 and gain of FLI1 expression during thrombocyte maturation. Blood Cells Mol Dis. 2010;44(3):175-180

40. Staley EM, Cao W, Pham HP, et al. Clinical factors and biomarkers predict outcome in patients with immune-mediated thrombotic thrombocytopenic purpura. Haematologica. 2019;104(1):166-175.

41. Moake JL, Rudy CK, Troll JH, et al. Unusually large plasma factor VIII:von Willebrand factor multimers in chronic relapsing thrombotic thrombocytopenic purpura. $N$ Engl J Med. 1982;307(23):1432-1435

42. Michels A, Albanez S, Mewburn J, et al. Histones link inflammation and thrombosis through the induction of Weibel-Palade body exocytosis. I Thromb Haemost. 2016:14(11):2274-2286

43. Fuchs TA, Bhandari AA, Wagner DD
Histones induce rapid and profound thrombocytopenia in mice. Blood. 2011;118 (13):3708-3714

44. Iyer N, Tcheuyap VT, Schneider S, Marshall $\mathrm{V}$, Jagadeeswaran P. Knockout of von Willebrand factor in zebrafish by CRISPR/Cas9 mutagenesis. Br J Haematol. 2019;186(4):e76-e80.

45. Zhang L, Lawson HL, Harish VC, et al Creation of a recombinant peptide substrate for fluorescence resonance energy transferbased protease assays. Anal Biochem. 2006;358(2):298-300

46. van Dorland HA, Mansouri Taleghani M Sakai K, et al. The International Hereditary Thrombotic Thrombocytopenic Purpura Registry: key findings at enrolment until 2017. Haematologica. 2019;104(10):21072115.

47. Semeraro F, Ammollo CT, Morrissey JH, et al. Extracellular histones promote thrombin generation through platelet-dependent mechanisms: involvement of platelet TLR2 and TLR4. Blood. 2011;118(7):1952-1961.

48. Mizuno T, Yoshioka K, Mizuno M, et al. Complement component 5 promotes lethal thrombosis. Sci Rep. 2017;7:42714. 